\title{
Site Unblinding Occurrence
}

National Cancer Institute

\section{Source}

National Cancer Institute. Site Unblinding Occurrence. NCI Thesaurus. Code C83058.

An indication or description that the trial site has been unblinded in a blinded clinical trial. 